\title{
Development and Validation of an Occupant Biomechanical Model for the Aortic Injury Analysis under Side Impacts
}

\author{
Ma Zhengwei ${ }^{1,2}$, Jing Lele ${ }^{2}$ and Jiang Lianbo ${ }^{2}$ \\ ${ }^{1}$ College of Mechatronics and Control Engineering, Shenzhen University, Shenzhen 518060, Guangdong, P. R. China \\ ${ }^{2}$ College of Urban Transportation and Logistics, Shenzhen Technology University, Shenzhen 518118, Guangdong, P. R. China
}

\begin{abstract}
Traumatic rupture of the aorta (TRA) is one of the leading causes of death in side impacts. However, the injury mechanism of TRA is still not clear now. In this study, an occupant biomechanical model for the aortic injury study was presented. The anatomical structures and mechanical characteristics of the thoracic organs, especially the cardiac aortic system, were replicated as precise as possible. Through model validations against the Post Mortem Human Subjects (PMHS) tests, good agreements were achieved between them in terms of the aortic strain, stress and deflection responses and injury distributions. Moreover, it was found that the injury mechanisms of the aorta under pure left side impact and oblique left side impact were different. In pure left side impact, the peri-isthmic region and descending aorta presented higher risks of TRA. In oblique left side impact, the TRA risk in aortic boot was higher than in other regions. The biomechanical model presented in this study could be of use to both the injury mechanism study of TRA as well as the design of occupants' safety countermeasures involving aortic injuries in side impacts.
\end{abstract}

\section{Introduction}

Side impacts are a particularly dangerous form of vehicle collisions and usually lead to serious casualties. In side impacts, traumatic rupture of the aorta (TRA), though infrequent, is one of the leading causes of occupants' fatalities. It has been reported that TRA occurs in only $1.2 \%$ of all accidents, but accounts for $21.4 \%$ of all fatalities. The incidence of TRA is found twice higher in side impacts $(2.4 \%)$ than that in frontal ones (1.1\%)[1]. The aorta is a key organ supplying blood to the whole human body. Any ruptures of the aortic wall can lead to extensive blood loss, hemorrhagic shock, and death.

Given the life-threatening nature of the aortic injuries, lots of efforts have been made to investigate the occurrence conditions and injury mechanism of TRA. The most common method used by peers is analysis of in-depth and real-world crash databases. Fitzharris et al. (2004) reviewed in-depth crash data for TRA in the National Automotive Sampling System (NASS) Crashworthiness Data System (CDS) and the Cooperative Crash Injury Study (CCIS) database and concluded that TRA was more common in lateral impacts than frontal impacts[2]. The studies conducted by Richens et al. (2003)[3], Franklyn et al. (2003)[4], Burkhart et al. (2001)[5] also found similar phenomenon. Digges et al. (2011)[6] analyzed a lot of autopsy reports involving TRA and found that TRA occurred in three primary locations in vehicle collisions: the ascending aorta, the aortic root, and the descending aorta (Fig. 1). The aortic wall typically ruptures transversely. Initially, the tear primarily appeared in the internal layer (the intima). And then, it extended to the middle layer (the media) and the outermost layer (the adventitia). McGwin et al. (2003)[7, 8] and Bertrand et al. (2008)[1] reported that TRA injury risk increased with occupants' age, the delta- $V$ and intrusion of crashes, and decreased with the distance between seat and the impact point.

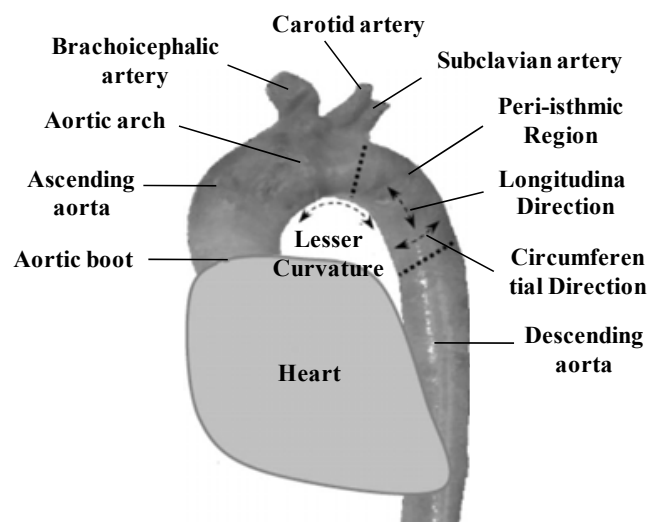

Fig. 1. Anatomical structures of the aorta.

The advancement of computer-aided techniques provides opportunity to study the aortic injuries by numerical methods. Some researchers developed models of isolated aorta, part or full human body to investigate the injury mechanisms of occupants' aorta in side impacts[9-16]. In 2001, Shah et al. improved a thorax model developed by predecessor to study the aortic injury[9]. And then, Shah et al. (2005)[10] integrated the improved thorax model with the abdomen model and the shoulder model developed by other researchers to build 
the first version of the Wayne State Human Body Model (WSHM). This model included main organs of the thorax, such as the heart, the lung and the aorta. With this model, Shah et al. (2005)[10] simulated the aortic responses under real accident conditions and studied the aortic injury mechanism. Some researchers, such as Siegel et al. (2010)[11] and Belwadi et al. (2012)[12], utilized the WSHM to investigated TRA in side collisions and proposed some protective countermeasures. Some numerical simulations about the isolated aorta are as follows. Richens et al. (2004)[13] developed a finite element (FE) model of the aorta and investigated its behavior during blunt traumatic impacts. Lee et al. (2007)[14] and Crosetto et al. (2011)[15] modeled the fluid-structure interactions of the cardiac aortic system. Maddali and Shah et al. (2005)[16] simulated the material and mechanical characteristics of the aorta under high speed stretching. In the study above[9-16], the injury mechanism of the aorta could be concluded as three types: the elastic injury, the viscous injury and the inertia injury. Both the deflection of the chest, the transmission of pressure wave, the inertia of the heart, and the stretching and tearing of the aorta contributed to TRA.

This study was aimed to present an occupant biomechanical model for the aortic injury study. Based on the Post Mortem Human Subjects (PMHS) tests performed by peers, several simulations were carried out to evaluate its biofidelity for predicting the aortic injuries in side impacts. Meanwhile, the aortic responses and injuries under pure left side impact and oblique left side impact were discussed.

\section{Method}

\subsection{Model description}

In previous study[17], an occupant biomechanical FE model of the Chinese 50th percentile male was developed. And the biofidelities of the full human body model and different body parts were evaluated. Fig. 2 shows the full human body FE model of occupants. The model was developed based on the the medical image data of the real human body. It included details of the skull, brain, skeleton, internal organs, skin, muscles, ligaments, main arteries and veins, and other tissues. Details about the model development, material modeling and biofidelity evaluations could be seen in reference 17 . This study mainly focuses the modeling of the thoracic aorta and its biofidelity for predicting the aortic injuries in side impacts. Fig. 3 and Fig. 4 respectively show the modeling of the thoracic organs and the cardiac aortic system. It can be seen that the anatomical structures of the thoracic organs, especially the cardiac aortic system, were replicated as precise as possible. The internal organs, such as the heart and lungs, were modeled with viscoelastic solid elements, while the aorta and veins were modeled with elastic shell elements. The blood inside the ventricles, arteries and veins, was simulated with elastic-fluid solid elements. A contact algorithm of ALE (Arbitrary Lagrangian Eulerian) in LS-DYNA 971 was defined between them. Table 1 lists the material parameters of the main thoracic tissues and organs.

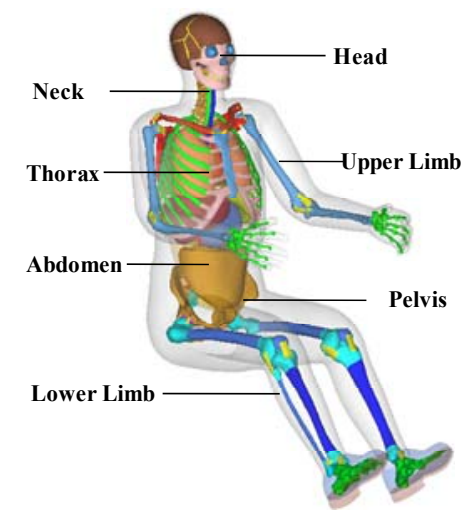

Fig. 2. Full human body FE model.

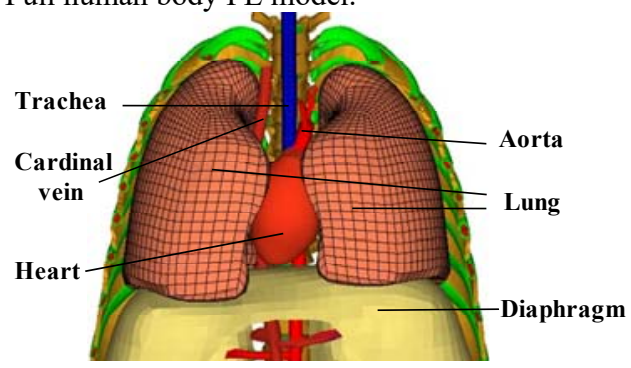

Fig. 3. The thoracic organs' model.

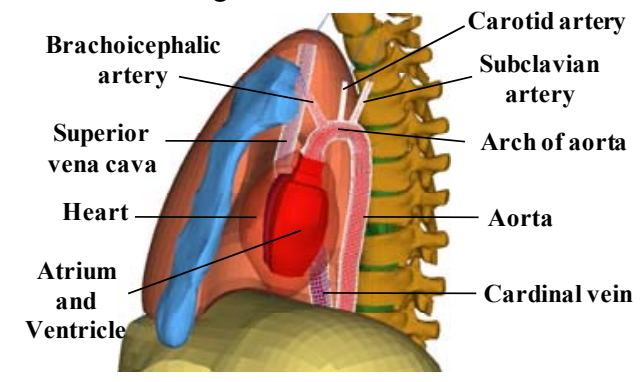

Fig. 4. Modeling of the cardiac aortic system.

Table 1. Material properties for the thoracic internal organs.

\begin{tabular}{llccccccc}
\hline \multicolumn{1}{c}{ Tissues } & Material Model & $\rho\left(\mathrm{kg} / \mathrm{mm}^{3}\right)$ & $K(\mathrm{Gpa})$ & $E(\mathrm{GPa})$ & $\mu$ & $G_{0}(\mathrm{Gpa})$ & $G_{\infty}(\mathrm{Gpa})$ & $\beta$ \\
\hline Heart & Viscoelastic & $1.00 \mathrm{E}-06$ & $2.60 \mathrm{E}-03$ & - & - & $4.40 \mathrm{E}-04$ & $1.50 \mathrm{E}-04$ & $2.50 \mathrm{E}-01$ \\
Lung & Viscoelastic & $6.00 \mathrm{E}-07$ & $2.20 \mathrm{E}-04$ & - & - & $2.00 \mathrm{E}-05$ & $7.50 \mathrm{E}-05$ & $2.50 \mathrm{E}-01$ \\
Diaphragm & Elastic & $1.00 \mathrm{E}-06$ & - & $1.55 \mathrm{E}-01$ & 0.40 & - & - & - \\
Trachea & Elastic & $1.20 \mathrm{E}-06$ & - & $1.70 \mathrm{E}-02$ & 0.40 & - & - & - \\
Aorta & Elastic & $1.20 \mathrm{E}-06$ & - & $5.00 \mathrm{E}-03$ & 0.40 & - & - & - \\
Vena & Elastic & $1.20 \mathrm{E}-06$ & - & $2.00 \mathrm{E}-02$ & 0.40 & - & - & - \\
Blood & Elastic-Fluid & $1.00 \mathrm{E}-06$ & $2.20 \mathrm{E}+00$ & - & 0.45 & - & - & - \\
\hline
\end{tabular}


Note- $\rho$ : Density, $K$ : Bulk Modulus, $E$ : Young's Modulus, $\mu$ : Poission's Ratio, $G_{0}$ : Short-term Shear Modulus, $G_{\infty}$ : Long-term Shear Modulus, $\beta$ : Decay Factor. The $K, E$ and $\mu$ satisfy the following relation: $K=E / 3(1-2 \mu)$.

\subsection{Model validation}

\subsubsection{PMHS tests}

From 2006 to 2008, Hardy et al. conducted a series of quasi-static and dynamic PMHS tests to investigate TRA mechanisms $[18,19]$. The pure left side impact test (Test XR5) and oblique left side impact test (Test XR8) of Hardy et al. (2008) [19] were selected for model validation in this study. In the tests, the lower extremities of all the PMHS were amputated. Meanwhile, all the PMHS were prepared using an axillary approach to place radiopaque markers on the aorta in the peri-isthmic region, which was shown in Fig. 5. The markers were used for subsequent imaging by the high-speed, biplane $\mathrm{x}$-ray to examine the general motion of the aorta within the mediastinum, and to measure strain in the periisthmic region of the aorta during impacts. Since the muscles and internal organs of PMHS lose the activity characteristics of living human body, the heart location of PMHS is typically more caudal and dorsal as compared to in the human. The heart also tends to pitch rearward which makes it very difficult to generate tension in the peri-isthmic region of PMHS aorta during impacts. To compensate these defects, the tests were conducted with the PMHS inverted, and angled such that the mediastinal contents assumed more anatomically correct positions. The PMHS were suspended and positioned using a network of straps and ratchet mechanisms. In addition, a pump perfusion pressure of about $7 \mathrm{kPa}$ was supplied continuously to the cardiac aortic system and respiratory system during impacts to simulate the physiologic state of living human body. The tests were carried out utilizing a $32-\mathrm{kg}$ impactor with a 152-mm diameter, 200-mm long polyethylene probe. The probe provided a circular flat-face impact surface. The impactor was accelerated up to a specified speed and then allowed to move freely into the subject up to a specified penetration.

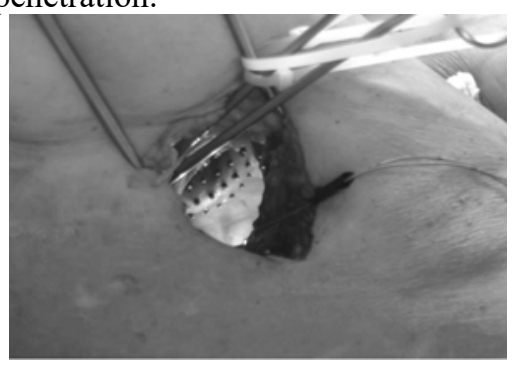

Fig. 5. The axillary approach used for PMHS.

For the pure left side impact test (Test XR5), the PMHS was inverted, pitched rearward 30-degrees from vertical. The test configuration can be seen in Fig. 6 . The upper extremities were allowed to dangle below the PMHS, so that the ribs were engaged by the impactor on the left side and fixed support plate on the opposite side.
The impactor was accelerated up to a velocity of $7 \mathrm{~m} / \mathrm{s}$ to impact the left side of the PMHS. The center of the impactor was aligned at the same vertical height as the xiphoid process, while the impact direction was through the center-of-gravity (CG) of the torso to minimize rotation. The penetration was limited to $104 \mathrm{~mm}$. In this test, the markers were also placed on the surface of the spine and sternum to examine their motions during impact.

For the oblique left side impact test (Test XR8), the PMHS was first inverted, pitched rearward 40 degrees from vertical, and then rotated 30 degrees in the global horizontal plane. The test configuration is shown in Fig. 7. The spine was fixed on the support beam of the test fixture, and the upper extremities were allowed to dangle below the PMHS. This configuration directed the impactor toward the spine through the lower left ribs, slightly to the left of the sternum. The center of the impactor was located at the same vertical height as the xiphoid process too. The PMHS was impacted at a velocity of $7 \mathrm{~m} / \mathrm{s}$. The penetration was limited to 136 $\mathrm{mm}$.

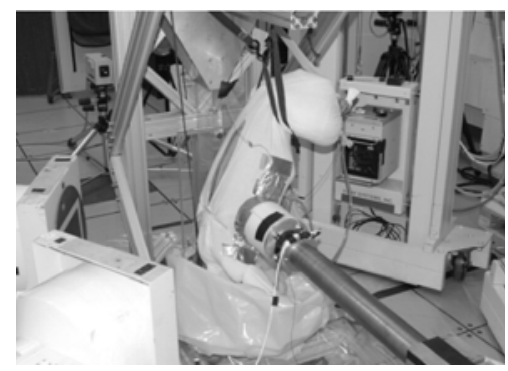

Fig. 6. Pure left side impact test (Test XR5) configuration.

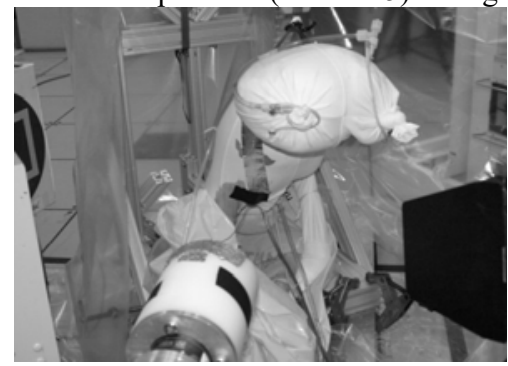

Fig. 7. Oblique left side impact test (Test XR8) configuration.

\subsubsection{Model simulation configurations}

The model validations were performed based on the tests described above. Fig. 8 (a) and Fig. 8 (b) respectively show the simulation set-ups against the pure left side impact test and the oblique left side impact test. The simulation set-ups were displayed in front for a convenience of observation. In model validations, both the upper and lower extremities were removed. A pressure of $7 \mathrm{kPa}$ was defined in advance for the cardiac aortic system and respiratory system. The initial velocity of the impactor was set as $7 \mathrm{~m} / \mathrm{s}$, with the penetrations being defined as same as in the tests. Other boundary 
conditions of the simulations were also consistent with the experiments.

The validations were conducted by comparing the model's responses, such as the peak impact force, aortic peak stress, aortic strain response in peri-isthmic region, deflections and injury distributions, with those in the

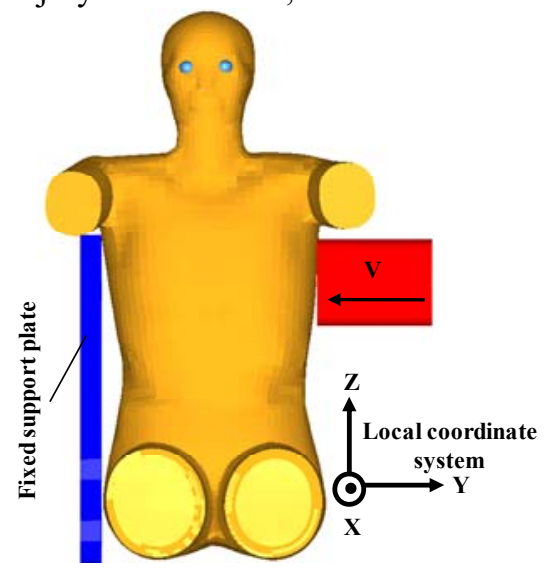

(a) Pure left side impact simulation

Fig. 8. Model simulation configurations against PMHS tests.

\section{Results and Discussion}

\subsection{Pure left side impact simulation}

From model simulation against the pure left side impact test (Test XR5), it could be seen that the thorax was impacted and reached its maximum deformation at about $30 \mathrm{~ms}$. Then the thoracic deformation recovered gradually. Fig. 9 (a) and Fig. 9 (b) respectively show the deformations and injury distributions of the thoracic rib cage and internal organs. Obviously, the rib cage sustained multiple rib fractures on the left and right sides. The internal organs, such as the left lung, spleen, aorta and diaphragm, presented serious contusions and tears with the injury degree of AIS 4+.

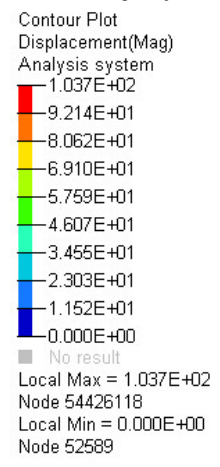

(a) Rib cage injury

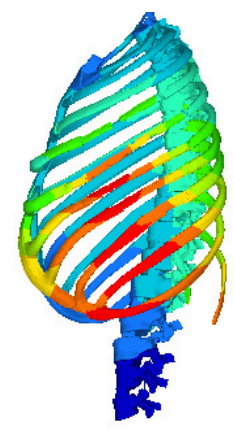

(b) Internal organs injury

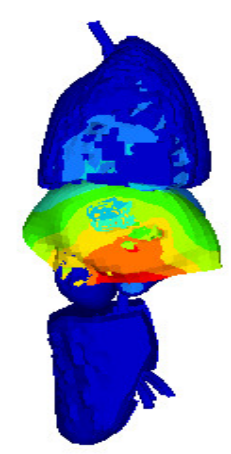

Fig. 9. Deformations and injury distributions of the thorax.

The aortic strain distribution at peak time is shown in Fig. 10. It could be seen the aortic peak strain lied in the peri-isthmic region and descending aorta. In model simulation, the peak strain of the peri-isthmic region was 0.148 , which was closed to the test results $(0.158)$. tests. Since the limited penetrations in the tests, the thoracic and aortic responses mainly occurred within 50 $\mathrm{ms}$. Therefore, the simulation time in model validations were set as $50 \mathrm{~ms}$ for the consideration of calculation time shortening.

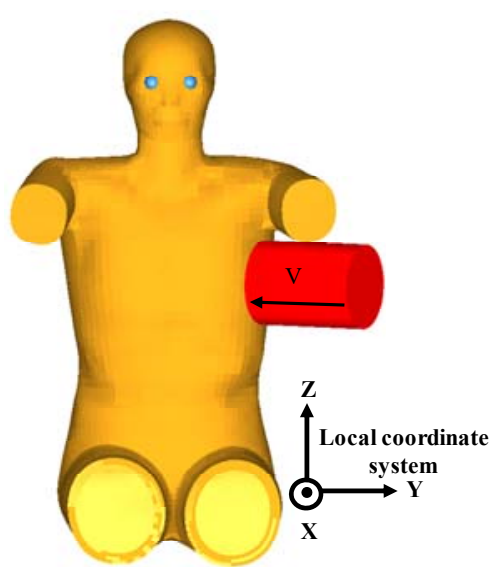

(b) Oblique left side impact simulation

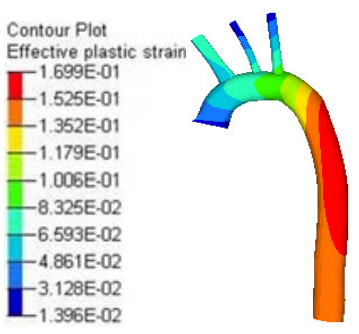

Fig. 10. Aortic strain distribution at peak time.

Fig. 11 shows the comparison of simulation and test responses, in terms of average longitudinal Lagrange strain time histories for the target locations associated with the greatest peak strain. It exhibited a good consistency in terms of the overall trend.

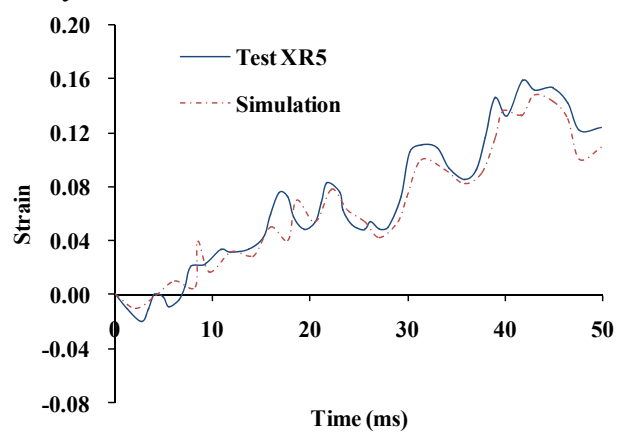

Fig. 11. Comparison of average longitudinal Lagrange strain time histories between simulation and test (Test XR8).

The comparisons of peak response values and rib fractures between simulation and test are listed in Table 2. With exception of the slight difference in rib fractures, the peak response values in simulation agreed well with the test results. All these comparisons above indicate that the occupant biomechanical model presented in this study is able to predict the aortic responses and injuries in pure side impact. 
Table 2. Comparison of the simulation and test results.

\begin{tabular}{|c|c|c|c|c|c|}
\hline \multirow{2}{*}{$\begin{array}{l}\text { Response } \\
\text { values }\end{array}$} & \multirow{2}{*}{$\begin{array}{c}\text { Peak } \\
\text { impact } \\
\text { force } \\
(\mathrm{kN})\end{array}$} & \multirow{2}{*}{$\begin{array}{c}\text { Peak } \\
\text { intraluminal } \\
\text { aortic stress } \\
(\mathrm{kPa})\end{array}$} & \multirow{2}{*}{$\begin{array}{l}\text { Peak } \\
\text { isthmic } \\
\text { strain }\end{array}$} & \multicolumn{2}{|c|}{ Rib fractures } \\
\hline & & & & $\begin{array}{c}\text { Left } \\
(1-12)\end{array}$ & $\begin{array}{c}\text { Right (1- } \\
12)\end{array}$ \\
\hline Test XR8 & 3.17 & 40 & 0.158 & $1-7$ & $3-4 、 6-8$ \\
\hline Simulation & 3.11 & 40.6 & 0.148 & $3-9$ & $4-8 、 11$ \\
\hline
\end{tabular}

\subsection{Oblique left side impact simulation}

In model simulation against the oblique left side impact test (Test XR8), the thoracic maximum deformation also occurred at about $30 \mathrm{~ms}$. The general deformations and injuries of the thorax are shown in Fig. 12, where (a) and (b) correspond to the deformations and injury distributions of the thoracic rib cage and internal organs, respectively. It could be seen that both rib fractures and internal organs injury in oblique left side impact were more serious than in pure left side impact. The rib cage suffered from a wide range of fractures on both the left and right sides, which was collected in Table 3. The internal organs, such as the left and right lung, liver, spleen, kidney, aorta and diaphragm, sustained serious contusions and tears with the injury degree of AIS 5+.

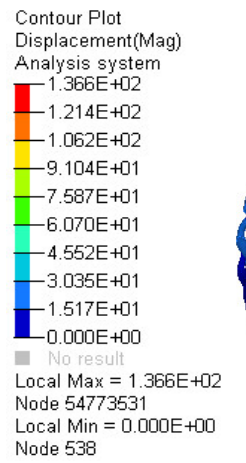

(a) Rib cage injury
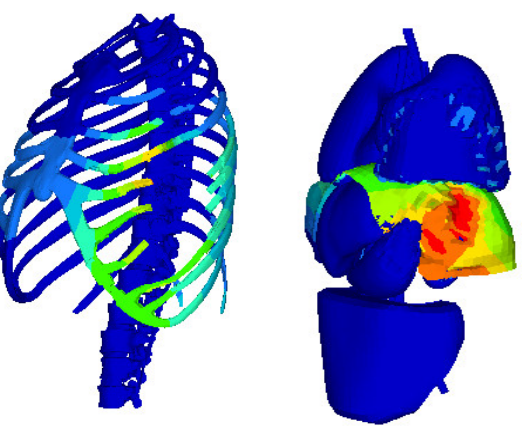

(b) Internal organs injury
Fig. 12. Deformations and injury distributions of the thorax.

Fig. 13 shows the aortic strain distribution at peak time. It could be seen the aortic peak strain located in the aortic boot with a value of 0.263 . Fig. 14 shows the comparison of simulation and test responses, in terms of average longitudinal Lagrange strain time histories for the target locations associated with the greatest peak strain. It exhibited a good consistency in terms of the overall trend.

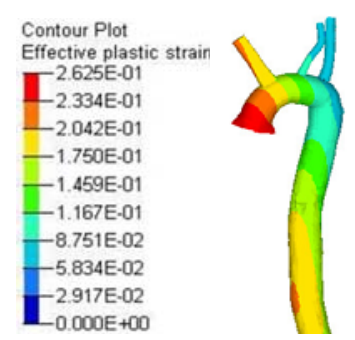

Fig. 13. Aortic strain distribution at peak time.

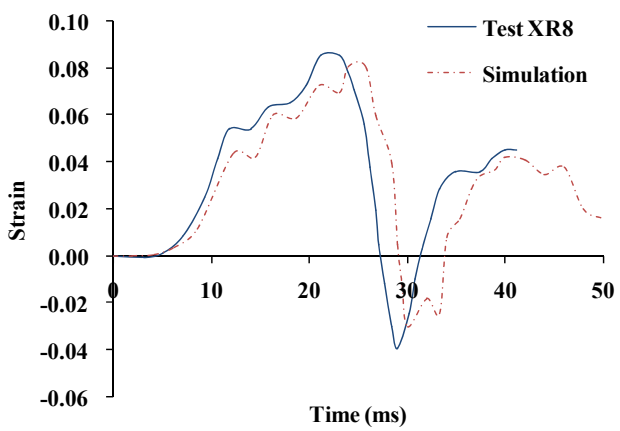

Fig. 14. Comparison of average longitudinal Lagrange strain time histories between simulation and test (Test XR8).

The comparisons of peak response values and rib fractures between simulation and test are listed in Table 3. Apparently, both rib fractures and the peak response values in simulation agreed well with the test results. All these comparisons above indicate that the occupant biomechanical model presented in this study is able to predict the aortic responses and injuries in oblique side impact.

Table 3. Comparison of the simulation and test results.

\begin{tabular}{cccccc}
\hline Response & Peak & Peak & Peak & \multicolumn{2}{c}{ Rib fractures } \\
values & $\begin{array}{c}\text { force } \\
\text { fon })\end{array}$ & $\begin{array}{c}\text { intraluminal } \\
\text { aortic stress } \\
(\mathrm{kPa})\end{array}$ & $\begin{array}{c}\text { Psthmic } \\
\text { strain }\end{array}$ & $\begin{array}{c}\text { Left } \\
(1-12)\end{array}$ & $\begin{array}{c}\text { Right } \\
(1-12)\end{array}$ \\
\hline Test XR8 & 5.29 & 54.7 & 0.087 & $2-10$ & $3-9$ \\
Simulation & 5.21 & 55.4 & 0.081 & $2-10 、 12$ & $4-9$ \\
\hline
\end{tabular}

\subsection{Injury mechanism}

From the simulations above, it was found that the injury mechanisms of the aorta under pure left side impact and oblique left side impact were different. It could be attributed to the aortic motions and deformations during impacts. Fig. 15 and Fig. 16 respectively show the aortic deformations in the pure left side impact and oblique left side impact. The wireframes depict the initial configurations of the aorta. The fringe plots show the deformation of the aorta at the time of peak longitudinal Lagrange tensile strain for the aorta.

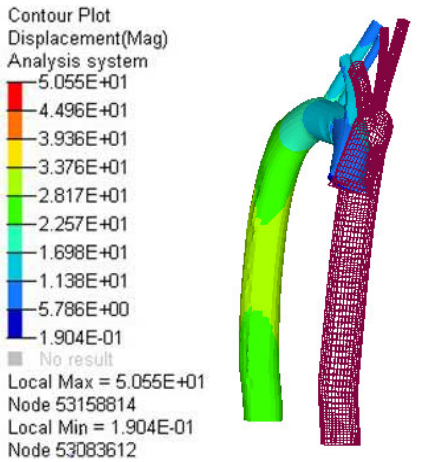

(a) Front view

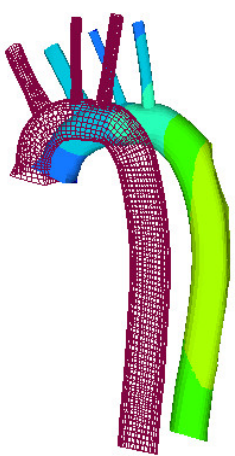

(b) Left view
Fig. 15. Aortic deformation in pure left side impact. 


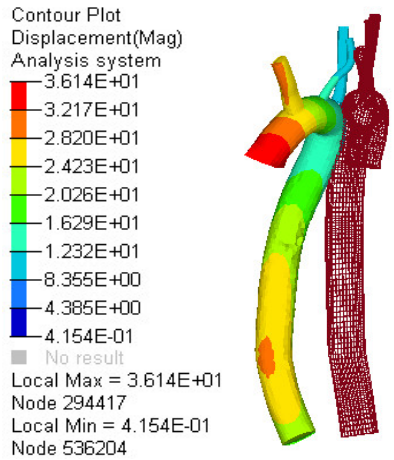

(a) Front view

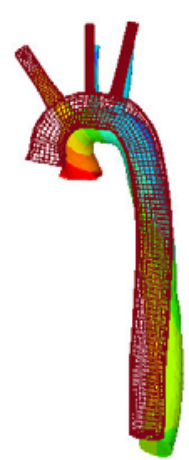

(b) Left view
Fig. 16. Aortic deformation in oblique left side impact.

In the pure left side impact, the aorta presented a large motion and deformation to right. Meanwhile, the anteroposterior expansion of the thoracic cavity resulted in an opposite motion between the heart and spine. This would make the aortic arch be straightened and lead a higher risk of TRA in the peri-isthmic region and descending aorta. In the oblique left side impact, the aorta was bended to right and the aortic arch was left related to the whole aorta. The movement of the heart to right and back would make the lesser curvature of the aorta be fold and the aortic boot be stretched excessively. This would result in a higher risk of TRA in the aortic boot.

\section{Conclusion}

In this study, an occupant biomechanical model for the aortic injury study was presented. The anatomical structures and mechanical characteristics of the thoracic organs, especially the cardiac aortic system, were replicated as precise as possible. Through model validations against the Post Mortem Human Subjects (PMHS) tests, good agreements were achieved between them in terms of the aortic strain, stress and deflection responses and injury distributions. It indicated that the biomechanical model presented in this study was able to predict the aortic responses and injuries in side impacts. Moreover, it was found that the injury mechanisms of the aorta under pure left side impact and oblique left side impact were different. In pure left side impact, the periisthmic region and descending aorta presented higher risks of TRA. In oblique left side impact, the TRA risk in aortic boot was higher than in other regions.

This study is supported by the Natural Science Foundation of Guangdong Province (Fund No. 2018A030310523), the Foundation for Distinguished Young Talents in Higher Education of Guangdong Province (Grant No. 2016KQNCX145), the Knowledge Innovation Program for Basic Research of Shenzhen City (Fund No. JCYJ20160520164316902), and the Research Projects of Shenzhen Technology University (Grant No. 1813303006008008 and 201716). The authors would like to thank the support of these projects.

\section{References}

1. Bertrand S., Cuny S., Petit P., et al. Traumatic Rupture of Thoracic Aorta in Real-World Motor Vehicle Crashes. Traffic Inj Prev. 9, 153-161 (2008)

2. Fitzharris M., Franklyn M., Frampton R., Yang K., Morris A., Fildes B. Thoracic Aortic Injury in Motor Vehicle Crashes: The Effect of Impact Direction, Side of Body Struck, and Seat Belt Use. J Trauma Acute Care. 57, 582-590 (2004)

3. Richens D., Kotidis K., Neale M., Oakley C., Fails A. Rupture of the Aorta Following Road Traffic Accidents in the United Kingdom 1992-1999: The Results of the Co-operative Crash Injury Study. Eur J of Cardio-thorac Sur. 23, 143-148 (2003)

4. Franklyn M., Fitzharris M., Fildes B., Yang K., Frampton R., Morris A. A Preliminary Analysis of Aortic Injuries in Lateral Impacts. Traffic Inj Pre, 4, 263-269 (2003)

5. Burkhart H.M., Gomez G.A., Jacobson L.E., Pless J.E. Fatal Blunt Aortic Injuries: A Review of 242 Autopsy Cases. J Trauma Acute Care. 50, 113-115 (2001)

6. Digges K., Augenstein J., Hardy W.N., Cavanaugh J., Jermakian J.S., Shah C. Measurement of Aortic Injuries in Lower Severity Near-Side Impacts. SAE Paper, 110265 (2011)

7. McGwin G., Metzger J., Moran S.G., Loring W.R. Occupant- and Collision-Related Risk Factors for Blunt Thoracic Aorta Injury. J Trauma Acute Care. 54, 655-662 (2003)

8. McGwin G., Reiff D.A., Moran S.G., Loring W.R. Incidence and Characteristics of Motor Vehicle Collision-Related Blunt Thoracic Aortic Injury According to Age. J Trauma Acute Care. 52, 859866 (2002)

9. Shah C.S., Yang K.H., Hardy W.N., Wang K.H., King A.I. Development of a Computer Model to Predict Aortic Rupture due to Impact Loading. Proceedings of the 45th Stapp Car Crash Conference, (2001)

10. Shah C.S., Maddali M., Mungikar S.A., Beillas P., Hardy W.N., Yang K.H., Digges K. Analysis of a Real-World Crash Using Finite Element Modeling to Examine Traumatic Rupture of the Aorta. 2005 SAE World Congress. (Detroit, MI, April, 2005)

11. Siegel J.H. Analysis of the Mechanism of Lateral Impact Aortic Isthmus Disruption in Real-Life Motor Vehicle Crashes Using a Computer-Based Finite Element Numeric Model: With Simulation of Prevention Strategies. J Trauma Acute Care. 68, 1375-1975 (2010)

12. Belwadi A., Siegel J.H., Singh A., Smith J.A., Yang K.H., King A.I. Finite Element Aortic Injury Reconstruction of Near Side Lateral Impacts Using Real World Crash Data. J Biomech Eng-T ASME. 134, 011006 (2012) 
13. Richens D., Field M., Hashim S., et al. A Finite Element Model of Blunt Traumatic Aortic Rupture. Eur J Cardio-thoracic Sur. 25,1039-1047 (2004)

14. Lee S.H., Kent R. Blood Flow and Fluid-Structure Interactions in the Human Aorta During Traumatic Rupture Conditions. Stapp Car Crash J. 51, 1-27 (2007)

15. Crosetto P., Reymond P., Deparis S., Kontaxatis D., Stergiopulos N. Fluid-Structure Interaction Simulation of Aortic Blood Flow. Comput Fluids. 43, 36-57 (2011)

16. Maddali M., Shah C.S., Yang K.H. Finite Element Modeling of Aortic Tissue Using High Speed Experimental Data. Proceedings of IMECE'05. (Orlando, FL, 2005)
17. Ma Z.W., Jing L.L., Lan F.C., Wang J.L., Chen J.Q. Development and Biofidelity Evaluation of an Occupant Biomechanical Model of a Chinese 50th Percentile Male for Side Impact. J Mech Med Biol. 17, 1740039 (2017)

18. Hardy W.N., Shah C.S., Kopacz J.M., et al. Study of Potential Mechanisms of Traumatic Rupture of the Aorta Using In-situ Experiments. Stapp Car Crash J. 50, 247-266 (2006)

19. Hardy W.N., Shah C.S., Mason M.J., et al. Mechanisms of Traumatic Rupture of the Aorta and Associated Peri-isthmic Motion and Deformation. Stapp Car Crash J. 52, 233-265 (2008) 